\title{
XXVIII.
}

Aus dem pharmakologisehen Institut der Universität Leipzig.

\section{Ueber die Wirkungen des Giftes der Larven ron Diamphidia locusta (Pfeilgift der Kalachari).}

Von

Dr. med. Franz Starcke.

Der Aufforderung des Herm Prof. R. Boehm folgend, babe ich im Leipziger pharmakologischen Institut die Wirkungen des von den Buschmännern der Kalachari als Pfeilgift gebrauchten Giftes der Käferlarve von Diamphidia locustal) auf verschiedene Thiere und die durch die Vergiftung in den Organen hervorgerufenen pathologischen Veränderungen genauer untersucht.

Die Giftlösung wurde durch 24 stündige Maceration der Larven mit durch etwas Chloroform sterilisirtem Wasser (1 cem Wasser auf 1 Larve) hergestellt. In der Regel kam die am stärksten wirkende erste Maceration, zuweilen da, wo es auf kleinere Dosen ankam, auch die zweite Maceration zur Verwendung.

Die Einverleibung des Giftes geschah unter aseptisehen Cantelen unter die Haut, in die Peritonealhöhle oder in eine Vene.

Als Versuchsthier dienten Frösche, ein Sperling, Tauben, ein Hahn, Kaninehen, Katzen und Hunde.

- Zum Zwecke der histologischen Untersuchung wurde neben der Fixirung in Alkohol und M üll e r'scher Flüssigkeit die in concentrirter wässriger Sublimatlösung, in Hermann'scher Lösung (Platinchlorid-OsmiumEisessig) und die Altmann'sche Lösung (Kaliumbichromat-Osmium) bevorzugt. Zur Färbung benutzte ich bei den Sublimatpräparaten Delafieldsches Hämatoxylin und wässriges Eosin, bei den anderen das Altmannsche Anilin-Säurefuchsingemisch mit Pikrinsäuredifferenzirung. Besonders scharfe Bilder ergab die bisher wenig angewandte Combination der Hermann'schen Fixirung mit der Altman n'schen Färbung. Zur Einbettang: wurde ein Gemisch von 52 und $58^{\circ}$ Paraffin benutzt. Die Dicke der Schnitte betrug $7,5-1,5 \mu$.

1) Vgl. die vorhergehende Abhandlung. 
Ueber die Wirkungen des Giftes der Larven von Diamphidia locusta. 429

1. Verlauf und Symptome der Vergiftung.

Ich beginne mit der Schilderung des Vergiftungsbildes bei Sä ug e thieren.

Bald nach der Injection von $0,5-1,0 \mathrm{ccm}$ einer wirksamen Lösung unter die Rückenhaut, je nach der Dosengrösse früher oder später hören Kaninchen auf zu fressen, verlieren nach und nach ihre Munterkeit und bleiben, ohne dass auffallende Veränderungen der Respiration und Herzthätigkeit nachweisbar sind, ruhig und in normaler Körperhaltung in einer Ecke des Käfigs sitzen. Nach Verlauf von 4-10 Stunden findet man in der Regel eine tief blutroth gefärbte Harnentleerung vor, und die abgesetzten Faeces werden weich und zuweilen ziemlich copiös. In sehr acut ablaufenden Fällen fällt das Kaninchen, ohne dass vorher irgend ein auffallenderes Symptom sich zeigt, frühestens nach 7 Stunden auf die Seite, vermag den Kopf nicht mehr zu heben und verendet dann binnen Kurzem unter schwachen Zuckungen und allmählichem Erlöschen der Athmung. In einigen Fällen gingen dem Tode auch ausgesprochene nervöse Symptome, starke Herabsetzung der Sensibilität und 2 mal heftige allgemeine Convulsionen voraus.

Mit kleinen Dosen vergiftete Kaninchen können viele Tage am Leben bleiben und sich wohl auch gelegentlich ganz von der Wirkung des Giftes wieder erbolen. Die dann etwas später, oft erst am zweiten Tage bemerkbaren Symptome sind die gleichen: Aufhören der Nahrungsaufnahme, blutrother Harn und weiche Darmentleerungen. Vom 5.-4. Tage an wird dann der Harn wieder normal, und kehrt allmählich die Fresslust zurück. Trotzdem aber magern in der Folge die Thiere häufig stark ab. An der Injectionsstelle fallen in Biischeln die Haare aus, und darunter kommt dann in ziemlich weiter Ausdehnung nicht mebr verschiebbare dunkelbraune, pergamentartig eingetrocknete Haut zum Vorschein. In einem Falle löste sich beim Anfassen der gesträubten Haare die Epidermis mit den Haaren wie eine Schale $a b$; darunter zeigte sich als feuchte, nicht blutige Oberfläche das Corium, das nach einiger Zeit pergamentartig eintrocknete. Wiederholt sind solche scheinbar genesene Thiere doch noch nach 8-14 Tagen unter progressiver Abmagerung zu Grunde gegangen.

Bei der Untersuchung des Harnes wurden unveränderte rothe Blutkörperchen nicht gefunden; statt dessen aber viel feinkörnige Detritusmassen, zuweilen in Form von Cylindern angeordnet, die in haardünne, lange Schwänze ausliefen. Auch freie Epithelien, Epithelialeylinder und Tripelphosphatkrystalle kommen vor. Im Spectroskop liess der blutig tingirte Harn stets nur die Absorptionsstreifen 
des Oxyhämoglobins, nach der Reduction diejenigen des Hämoglobins, niemals aber eine Andeutung des Methämoglobinspectrums erkennen. Eiweiss war reichlich vorhanden, solange die abnorme Färbung bestand.

Genanere Blutuntersuchungen intra vitam sind noch nicht ausgeführt worden. In einem Versuche gaben während des ersten und zweiten Tages dem Ohre entnommene Blatproben ganz normale mikroskopische Bilder. Erst am dritten Tage zeigte sich eine auffallende Zunahme der Leukocyten und das Auftreten farbloser Scbollen und feinkörnigen Matriales (das betreffende Thier starb erst am 5. Tage).

Auch bei Hunden und Katzen war der Verlanf der Vergiftung niemals durch stürmische Erscheinungen ausgezeichnet und erstreckte sich nach subcutaner Einverleibung des Giftes je nach der Grösse der Dosis auf einen bis mehrere Tage. Selbst nach der in travenösen Injection war der Tod eines Hundes nach $61 / 2$ Stunden noch nicht erfolgt.

Als erste Zeichen der Wirkung bemerkt man auch bei diesen Thieren Abnahme der Munterkeit und die Entleerung blatig- und ikterisch gefärbten Harnes. Bei Katzen können schon nach 1 bis 21/2 Stunden paretische Erscheinungen in den hinteren Extremitäten sich einstellen. In acut, d. h. innerhalb 24 Stunden tödtlich endenden Fällen wurde keine Temperatursteigerung wahrgenommen; auch Erbrechen und Durchfälle fehlten. Dagegen entwickelte sich als charakteristisches Symptom allmählich im Laufe einiger Stunden eine zur completen Reactionslosigkeit fübrende Abnahme der Sensibilität. Die Thiere (Hund in gleicher Weise wie Katze) reagiren auf stark schmerzhafte Einwirkungen, tiefe Nadelstiche, Quetschung der Pfoten a. dgl. nicht mehr; auch der Cornealreflex fehlt; unter fortschreitender allgemeiner Lähmung erfolgt schliesslich der Tod.

Bei langsamerem Verlauf entstanden umfangreiche Eiterherde unter der Haut. Bei einem grossen, 25 Kilo schweren Hunde hatten sich 5 Tage nach der Injection an verschiedenen Stellen des Bauches und des Rückens acht grosse Abscesse gebildet, von denen mehrere spontan sich öffneten und in grosse eiternde Geschwüre sich verwandelten. In diesem Stadium stieg die Körpertemperatur des Thieres bis auf $41,3^{\circ} \mathrm{C}$. Ikterische Färbung, besonders an der Conjunctiva konnte sowohl in diesem wie auch in anderen langsamer verlaufenden Fällen sicher wahrgenommen werden.

Der Harubefund war derselbe wie bei Kaninchen: reichliche Mengen von Eiweiss und normalem Blutfarbstoff, k e in e unveränderten rothen Blutkörperchen, rothes, flockiges Sediment, bestehend aus 
Tropfen von rothgelber Fàrbe zu unregelmässigen, theils gewundenen Schnitren verklebt. Leukocyten und Epithelialcylinder fehlten.

Von Vögeln sind mit Tauben, einem Sperling und einem

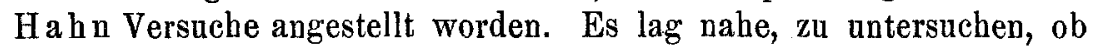
die Aufnahme der Larven per os, bei diesen Thieren Vergiftungserscheinungen hervorruft; ob die Larve vielleicht durch ihre Giftigkeit den Vögeln gegenüber geschützt ist, oder ob das Gift im Verdauungsapparat unschädlich gemacht wird. Das Letztere ist offenbar zutreffend. Ein mit einer Larve gefütterter Sperling blieb am Leben. Einer jungen aber ausgewachsenen Taube wurde eine Larve, welche 16 Stunden in Wasser gelegen hatte, zusammen mit der Macerationsflitssigkeit per os beigebracht. Das Thier verhielt sich darauf zwar ziemlich ruhig, zeigte aber, abgesehen von der Entleerung schwärzlich-blutig gefärbter Faeces keine krankhaften Symptome: 24 Stunden später erhielt dasselbe Thier noch drei trockene Larven per os, blieb aber auch hiernach munter und gesund.

Das Gift entfaltet seine tödtlichen Wirkungen auch bei Vögeln, wenn es unter die Haut gespritzt wird. Obige Taube starb infolge der Injection von 0,5 cem einer frischen Giftlösung unter die Brusthaut schon nach 70 Minuten. Eine Stunde lang nach der Vergiftung verbielt sie sich ruhig, fiel dann unter unbeholfenen krampfhaften Flïgelschlägen auf die Seite und verendete langsam. In ähnlicher Weise, nur etwas langsamer, verliefen auch einige andere an Tauben angestellte Versuche.

Frösche reagiren auf das Larvengift sehr langsam und träge; das Nähere ist aus beigefügtem Versucbsprotokoll ersichtlich.

Eine grosse Esculenta erhielt 1 ccm der Giftlösung in den Lymphsack. Während der ersten 2 Tage ist kein Zeichen einer toxischen Wirkung zu bemerken; aus der Blase abgepresster Harn ist klar und farblos Vom dritten Tage an findet eine allmähliche Zunahme des Körpervolumens statt; die Maulirommeln füllen sich mit röthlicher Flüssigkeit, und die Beweglichkeit des Thieres nimmt stetig ab. Am 5. Tage Abends lebt es noch: am 6. Morgens wird es todt gefunden.

Beim Eröffnen der Hautlymphsäcke fliesst viel hellblutiges Transsudat $\mathrm{ab}$, welches bald flockige Gerinnsel absetzt. An der Injectionsstelle starke hämorrhagische Färbung in der Ausdehnung eines Zehnpfennigstïckes sowohl an der Haut als auch an den Muskeln des Thorax und Halses. Die Innenseite der Hant in ihrer ganzen Ausdehnung ziemlich stark injicirt.

Bei der Eröffnung der Bauchhöhle fliesst ebenfalls blutrothes Trans: sudat ab. Der ganze Verdaungskanal ist oberflächlich tief dunkelroth gefärbt bis an die Mitte des Magens. Im Inneren des Magens nur blutig gefärbter Schleim; die Schleimhaut hell; im ganzen Darmkanal aber ist 
die Schleimhaut dunkelroth; mit der Lupe erkennt man nach Abspülung des blutigen Schleimes die Falten der Mucosa auf der Höhe sehr geröthet, stellenweise punkt- bis hirsekorngrosse Blutungen. Im Duodenum starke Ausdehnung und Injection der Zotteneapillaren. Der eine Hoden schwarz-roth hämorrhagisch gefärbt, der andere normal.

Ueberblickt man die nach der Vergiftung mit dem Larvengifte während des Lebens auftretenden Erscheinungen, so vermisst man, abgesehen von der constant bei Säugethieren beobachteten Hämoglobinurie Symptome, die auf ein bestimmtes Organ als Angriffspunkt der Wirkung des Giftes hindeuten könnten. Anderen Intoxicationen mit Thiergiften, z. B. dem Schlangengifte gegenüber, fällt besonders der auch nach intravenöser Einverleibung verhältnissmässig langsame Verlauf der Vergiftung anf, in deren Wesen wir erst durch das Stadium der pathologischen Veränderungen einen Einblick gewinnen, welche in verschiedenen Organen post mortem nachgewiesen werden können.

\section{Die pathologischen Befunde.}

In erster Linie fesseln die Veränderungen unsere Aufmerksamkeit, welche nach subcutaner Injection des Larvengiftes an der Haut, im subcutanen Zellgewebe und den angrenzenden Fascien, Muskeln und serösen Höhlen sich darbieten. Sie sind bei Kaninchen und Hunden in gleich hohem, bei Katzen in etwas geringerem Grade vorgefunden worden.

Dass schon während des Lebens in langsamer ablaufenden Vergiftungsfällen bei Kaninchen Hautveränderungen an der Injectionsstelle eintreten, ist oben schon angegeben worden. Auch bei Hunden ist gelegentlich schon während des Lebens entfernt von der Injectionsstelle an dünner behaarten Stellen der Bauchhaut eine diffus blutrothe Färbung wahrzunehmen.

Löst man nun aber nach eingetretenem Tode die Haut vom Körper $a b$, so ist man überrascht von der weiten Ausdehnung, in welcher, von der Injectionsstelle ausgehend, die um- und anliegenden Theile verändert sind.

Je nach dem Charakter dieser Veränderungen kann man zwei Stadien unterscheiden.

1. die diffuse blutig-ödematöse Infiltration,

2. die eiterige Entziundung.

Die erstere findet sich in allen Fällen, die innerbalb einer kürzeren Frist von 12-48 Stunden zum Tode geführt haben, die letztere nur dann, wenn das lethale Ende 3-8 Tage nach der Vergiftung erfolgt ist. Um die Veränderungen gut übersehen zu können, wurde 
durch einen vom Unterkiefer bis zur Symphyse in der Mittellinie geführten Schnitt die Haut durehschnitten und dann im Zusammenhang vom Rumpf und den Extremitäten sorgfältig abpräparirt. Nach acutem Verlauf der Intoxication zeigt sich die Injectionsstelle (in der Regel fand der Einstich etwa in der Mitte des Riickens statt) von einem ovalen rothen Hof umgeben, an dessen Peripherie die Blutgefässe der Haut stark injicirt sind. Von da ausgehend erstreckt sich dann nach abwärts bis in die Beckengegend und die der hinteren Extremität oder nach aufwärts bis gegen das Ellbogengelenk der Injectionsseite, je nachdem weiter unten oder oben injicirt worden ist, stets aber zugleich nach vorn über den Brustkorb und das Abdomen, gegen die entgegengesetzte Körperhälfte sich abrundend und die Mittellinie etwas ïberschreitend eine gleichmässige, blutrothe Färbung der Haut. Aus dieser rothen Fläche hebt sich eine grössere, von oben nach unten verlaufende und in der Leistengegend aussen an den Inguinallymphdrüsen vorbeiziehende Hautvene durch ihre starke Blutfüllung scharf $a b$, während die correspondirende Hautvene der entgegengesetzten Seite wenig hervortritt. Die Lymphdrüsen der Achselhöhle und Leistengegend sind auf der Injectionsseite geschwollen und hänfig dunkelblutroth gefärbt.

Abgesehen von der diffusen Röthung erscheint die Haut gallertartig ödematös infiltrirt, an einzelnen Stellen wie mit dicken Lagen geronnener rother Gallerte begossen. Das ödematöse Zellgewebe lässt sich schichtweise abpräpariren, und darunter kommt dann die Innenseite der Cutis mit stark injicirtem Capillarnetz zum Vorschein. Man überzeugt sich leicht davon, dass die Rothfärbung des ödematösen Gewebes nicht von Blutungen herrührt, da das Mikroskop ausserhalb der Blutgefüsse nirgends rothe Blutkörperchen nachweisen lässt: Es handelt sich vielmehr um eine Durchtränkung der Gewebsmaschen mit einem durch aufgelösten Blutfarbstoff gleichmässig gefärbten Transsudat. Niemals ist das vom Körper abgelöste Fell des Thieres in seinem ganzen Querdurchmesser in der beschriebenen Weise verändert. Immer bleibt die der Injectionsstelle gegenüberliegende Hälfte desselben zum grossen Theile normal. Die der Haut anliegenden Muskeln und Fascien sind am stärksten in der unmittelbaren Umgebung der Injectionsstelle, etwas weniger in weiterer Entfernung: von derselben in Mitleidensehaft gezogen, ähnlich wie die Haut intensiv geröthet, in der Regel aber etwas weniger ödematös infiltrirt.

Obwohl der Einstich sorgfältig immer nur unter die Haut geschehen war, pflanzten sich doch wiederholt die Veränderungen, in 
die Tiefe gehend, durch die Muskeln und Fascien bis in die Brustoder Bauchhöhle fort.

War z. B. in der Nierengegend am Rücken injicirt worden, so zeigte das parietale Blatt des Peritoneums, von der dem Injectionsbezirk correspondirenden Stelle ansgehend, mehrmals auch der dem Peritoneum anliegende Theil der Nierenoberfläche starke Röthung. In solchen Fällen fanden sich auch in der Bauchhöble, seltener in der Pleura und dem Pericard wechselnde Mengen flissigen roth gefärbten Transsudats.

Nach längerer Lebensdauer verendete Thiere weisen an Stelle des Oedems entsprechend ausgedehnte Eiterherde auf. Aus dem $\mathrm{Ob}$ ductionsprotokoll eines am 8. Tage nach der Vergiftung verstorbenen Kaninchens können wir als in dieser Beziehung typisches Beispiel Folgendes hier anführen: Von der Injectionsstelle in der Mitte des Rückens erstreckt sich ein eitriger Entzündungsherd nach oben und links bis an die Seapula. Die ganze linke Hälfte des Brustkorbes ist in eine eiternde Fläche verwandelt, die aber über das Sternum nicht weit nach rechts hinübergreift. Die Eiterbildung ist an diesen, weit vom Injectionsorte entfernten Stellen viel massiger als in der unmittelbaren Umgebung der Injection. Der Herd an der Einstichstelle ist von einem Kranze stark gefüllter Venen eingefasst, die scharf abschneidend beim Eintritt in die eiternde Fläche versehwinden. Der Tiefe nach erstreckt sich am Brustkorb die Eiterung durch die Muskeln und Faseien hindurch bis auf die Pleura und das Peritoneum, welch' letzteres stark injicirt, feucht und mit den ersten Anfängen einer fibrinösen Exsudation bedeckt erscheint. Das perirenale Gewebe ist entzlindet und eitrig infiltrirt. An der Hant bildet der Eiter eine dicke, käsige Schwarte.

Wie es bekanntlich bei Kaninchen die Regel ist, waren weder in dem oben beschriebenen, noch auch in anderen Fällen flüssige Eiteransammlungen entstanden. Dieses war aber in holem Maasse bei Hunden der Fall, wo sich in dem von der Injectionsstelle sich ausbreitenden Entzündungsgebiete an vielen Stellen grosse Eiterbeulen entwickeln, die sich noch während des Lebens nach aussen entleeren können.

Ueber ähnliche Veränderungen, welche die subcutane Injection des Larvengiftes bei Tauben bervorruft, giebt das folgende Versuchsprotokoll näberen Aufsehluss.

Eine sebr kräftige und lebhafte Tanbe erbält früh $8 \mathrm{Ubr} 0,2 \mathrm{ccm}$ in die Brustmusculatur injicirt. Darauf tagsiiber allgemeine Mattigkeit, die aber gegen Abend wieder überwunden ist. An der Injectionsstelle 
hat sich eine kirschgrosse Anschwellung gebildet, welche in den nächsten Tagen zurückgeht und verhärtet. Nachdem das Thier 9 Tage lang normales Verhalten gezeigt hat, wird eine erneute Injection von $0,3 \mathrm{ccm}$ vorgenommen. Während der darauffolgenden Nacht dünnbreiige, stark gallig gefärbte Kothentleerungen. Nach 24 Stunden vermag die Taube nicht mehr auffufliegen, sie lässt soeben genommenes Wasser wieder aus dem Schnabel herauslaufen und kann nur mit Mühe sich auf den Füssen stehend erhalten. Um für die mikroskopische Untersuchung mög. lichst frische Präparate zu erhalten, wird sie am Abend des zweiten Tages getödtet.

Die Section zeigt an der Stelle der ersten Injection ungefähr markstückgross und einige Millimeter in die Tiefe gehend einen krustenartigen Schorf, dessen Umgebung erweicht ist. An der Stelle der zweiten Injection ist die Haut durch Eiterung abgehoben. Beim Abpräpariren zeigt sich das subcutane Bindegewebe in der ganzen Ausdehnung der Brustmusculatur öd ematös und schmutzig graugrünlich verfärbt. Die Brustmuskeln seibst zu circa $2 / 3$ ibrer Masse sind gleichfalls schnautzig graugrtin verfärbt erweicht und da, wo sie an die Hant angrenzen, gallertartig infiltrirt. Durch die Brustmuskeln gelegte Schnitte zeigen unter dem Mikroskop eine scharfe Begrenzung der Eiterherde; ein dichter Wall von Eiterkörperchen trennt die normalen Partien von den degenerirten, fast structurlosen Muskelfasern.

Wie die Hämoglobinurie während des Lebens zu den charakteristischen Symptomen der Vergiftung mit dem Larvengifte gehört, so zeigen auch von den inneren Organen die Nieren regelmässig die auffallendsten pathologisehen Veränderungen post mortem. Makroskopisch sind dieselben gewöhnlich nur durch eine abnorme, von Dunkelroth bis Blauschwarz variirende und von einer starken Hyperämie bedingten Färbung angedeutet. Nur einmal bei einer Katze fanden sich zwischen Rinde und Mark einige hämorrhagische Herde. In der Regel waren beide Nieren in gleichem Maasse verändert. Ausnahmsweise beschränkte sich die Affection auf eine Niere, während die andere fast normale Befunde ergab.

Die mikroskopische Untersuchung, die nur in denjenigen Fällen ausgefuibrt wurde, wo die Organe sofort nach dem Tode frisch zur Verwendung kommen konnten, ergab Veränderungen

1. im System der Blutcapillaren,

2. in allen Abschnitten des secernirenden Parenchyms, und zwar in überwiegendem Maasse in den Glomeruli, Tubuli contorti und den aufsteigenden Schenkeln der Hen l e'schen Schleifen. Die absteigenden Schenkel letzterer und besonders die Tubuli recti wichen meistens nur hinsichtlich ihres Lumeninhaltes von der Norm ab.

Uebereinstimmend mit dem makroskopischen Befund zeigte auch das Mikroskop eine beträchtliche Erweiterung und starke Füllung: 
der Blutcapillaren. Ihr Inhalt bestand, abgesehen von den immer in überwiegender Menge vorhandenen normalen Blutzellen, aus feinkörnigen, mit wässrigem Eosin wie rothe Blutkörperehen färbbaren Detritusmassen. In einzelnen Fällen wichen auch die rothen Blutscheiben darin von der Norm ab, dass sie bei normalen Umrissformen fein granulirt erschienen.

Im Lumen der Secretionswege der Nieren von den Bowman * schen Kapseln bis berab za den Tubuli recti fanden sich stets ungewöhnliche feste Abseheidungen, und zwar in reichlichster Menge, wenn die Vergiftung nach 8-24 Stunden zum Tode geführt hatte. Die Harnkanälchen der Niere eines Hundes, der nach 24 Stunden verstorben war (die Nieren waren sofort in Alkohol gebracht worden), fanden sich dicht angefüllt mit gut ausgebildeten, grossen prismatisehen Hämoglobinkrystallen. Bei Verwendung anderer Härtungsmittel und bei anderen Thieren ist dieser Befund niemals vorgekommen.

Wenn wir die in den verschiedenen Abscbnitten des Secretions. apparates vorkommenden Befunde etwas näher verfolgen, so bieten sich zunächst in den Glomeruli folgende Veränderungen dar.

Die Schlingen des Capillarknäuels füllen den Raum der Bow. man'schen Kapsel mehr oder weniger vollständig aus. Das Lumen der Sehlingen erfüllen neben rothen Blutkörperchen feinkörnige oder auch durchsichtige Massen. Das Schlingenepithel ist grossentheils erhalten, seine Kerne gut gefärbt, die Zellcontouren aber sehr undeutlich wahrzunehmen. Auch das Kapselepithel scheint der Hauptsache nach unverändert zu sein. Im Kampselraume, zwischen Wand und Gefässknäuel sind fast immer Abscheidungen nachweisbar; sie beschränken sich da, wo die Knäuel die Kapsel nahezu ausfüllen, auf kleinere Mengen der Kapselwand anhaftender feiner Körner, nehmen aber auch die Form der oft beschriebenen Halbmonde an und können in solcher Masse vorhanden sein, dass der Gefässknäuel durch sie auf die Hälfte des Kapselraumes zusammengedrängt wird, In einem besonders prägnant ausgebildeten Fall dieser Art, in der Niere einer Katze, die nach 36 Stunden zu Grunde gegangen war, enthielt fast jede einzelne Bowman'sche Kapsel kleinere oder grössere Mengen dieser Abscheidungen, die aus einer wenig körnigen Grundmasse mit vielen hellen Vacuolen and zwischengelagerten Scblingenepithelien bestand. Die aus den Glomerusschlingen ausgetretenen Ablagerungen waren in diesem Falle so massenhafte, dass vielfach die an die Kapsel sich ansschliessenden Abschnitte der Tubuli contorti reichlich auf das 3 fache des normalen Durchmessers ausgedehnt und dicht mit dem soeben beschriebenen Material angefullt erschienen. 
Die Tubuli contorti weisen in den verschiedenen Versuchen aber auch in ein und demselben Präparate verschiedene Grade der patholo- gischen Veränderung auf. Ganz normal werden sie niemals angetroffen, Strichelung und Stäbchenstructur liessen sich nur an sehr wenigen Stellen sehr undeutlich erkennen. Im Falle weniger weitgehender Läsion ist der wandständige Theil des Protoplasma erhalten, entweder sehr fein körnig oder mit kleinen Vacuolen durchsetzt; der centrale Theil des Protoplasma entbehrt des scharfen Saumes, ist unregelmässig zackig begrenzt, wie angefressen und birgt den wenig oder gar nicht veränderten, sehr häufig von einer hellen Zone umgebenen Kern. Das Lumen solcher Kanällchen kann auf kurze Strecken frei sein; in der Regel führt es ähnlichen körnigen Detritus, wie er sich in den Kapseln findet.

In weiter fortgeschrittenen Stadien ist das Protoplasma an der Wand der Kanälchen auf eine schmale, fein granulirte Zone reducirt. die Zellgrenzen an beiden Seiten durch in feine Linien geordnete Protoplasmakörnchen markirt, während die Begrenzung der Zellen nach dem Lumen hin völlig verwischt ist. Der Kern ist nach dem Lumen hin vorgerïckt, die Zelle in eine durchsichtige, von einer äusserst dünnen Protoplasmaschicht umgebene Blase verwandelt. Auf Querschnitten der Contorti bilden die Kerne einen engen Kreis, in dessen Centrum feine Körnchen das Lumen vollends ausfüllen; auf Längsschnitten begrenzen die Kerne reihenweise die zusammenhängenden Bänder der Inbaltsmasse.

Endlich sind die Epithelien oft streckenweise im Zusammenhang exfoliirt, so dass die Membrana propria des Kanälehens blossliegt, eine Läsion, die häufig auch in den Henle'schen Schleifen, nur selten in den Sammelröhren vorkommt.

Während ansserdem auch die Epithelien der aufsteigenden Schleifenschenkel mebr oder weniger von Vacuolenbildung und Rarefication des Protoplasma betroffen sind, erscheint die Epithelialbekleidung: der Tubuli recti und der Abflussröhren unverändert.

Hämoglobinabscheidungen finden sich natürlich in Form der wiederholt beschriebenen körnigen Massen in wechselnder Menge in allen Theilen des Organes; in den Tubuli recti gesellen sich zu ihnen auch zahlreiche Epithelzellen und freie Kerne, die aus den oberen Partien herabgeschwemmt sind.

Die Deutung des Nierenbefundes bietet keine Schwierigkeiten, wenn man sich die Schilderungen vor Augen führt, welche M arch and '),

1) Virchow's Archiv, Bd. LXXVII. 
Lebedeff $\left.f^{1}\right)$ u. A. von den feineren Veränderungen der Niere infolge von Hämoglobinurie entworfen haben, wie sie durch verschiedene chemische Agentien, chlorsaure Salze, Jod, Glycerin u. s. w. leicht experimentell bei Thieren hervorgerufen werden kann.

Es führt das Studium jener Beschreibungen zu der Einsicht, dass die durch das Larvengift bedingte Hämoglobinurie allein schon ausreicht, um die oben geschilderten Läsionen der Nieren hervorzubringen, dass es demnach zum mindesten zweifelhaft ist, ob das Larvengift selbst dabei direct betheiligt ist.

Die auffallend starke Veränderung der Glomeruli in dem oben näher beschriebenen Falle bietet insofern Interesse, als bei anderweitig bedingter Hämoglobinurie im Ganzen die Betheiligung der Glomeruli nach der Schilderung der Autoren eine viel geringfügigere gewesen ist. Sie weist darauf bin, dass die Wände der Schlingencapillaren für gelöstes Hämoglobin in hohem Grade durchlässig werden können, und es wäre möglich, dass diese Veränderung durch die Anwesenheit des Larvengiftes besonders begünstigt wird.

Nächst den Nieren findet man die stärksten pathologischen Veränderungen im Darmkanal, und zwar bei Hunden und Katzen. in ausgeprägterer Form als bei Kaninchen. Dass auch beim Frosch die Schleimhant des Intestinaltractus afficirt wird, ist aus dem oben (S. 432) mitgetheilten Versuchsprotokoll ersichtlich.

Blutige Darmentleerungen kamen bei Hunden and Katzen nicht vor, bei Kaninchen werden die Faeces bei längerer Versuchsdauer weich und breiig. Geringere Grade der Veränderung der Darmschleimhant bestehen in Hyperämie und reichlicher Auflagerung von Schleim und desquamirtem Zottenepithel. In den höheren Graden finden sich im Dünndarm schwarzrothe Massen, unter denen die Schleimhaut das Aussehen dunkelrothen Sammets darbietet, ähnlich verhielt sich bei Hunden und Katzen auch die Magenschleimhaut. Blutungen in der Intestinalmucosa fehlen oder sind nur in Form vereinzelter kleiner Echchymosen vorhanden. Mit der Lupe erkennt man in der stark byperämisehen Schleimhaut die starke Injection der Blutcapillaren als Ursache der intensiven Röthung. Durch das Mikroskop lässt sich die Desquamation des Zottenepithels in grossen zusammenhängenden Stïcken in weitester Ausdehnung constatiren. Die abgestossenen Epithelien sind mit dem in Darmlumen offenbar geronnenen hämoglobinhaltigen Transsudat innig verschmolzen. Die Zotten ragen nackt ins Darmlumen hinein. Exfoliation der Drüsenepithelien war nicht nachzuweisen.

1) Virchow's Archiv. Bd. XCI. 
Die Röthung kann sich auch auf die Darmserosa und das Peritoneum erstrecken, während in der Abdominalhöhle klares, aber rothgefärbtes Transsudat in wechselnden Mengen gefunden wird.

In allen übrigen Organen konnten trotz wiederholter sorgfältiger mikroskopischer Untersuchung prägnantere Befunde nicht ermittelt werden. Die Leber zeichnete sich häufig durch starke, capillare Hyperämie, insbesondere der Vena centralis der Acini aus, die Milz durch vergrössertes Volumen, dunkle Färbung und stärkere Anhänfung von Pigmentkörnern, in den Lungen, im Centralnervensystem, sowie auch in Herz und Muskeln liess die Vergiftung keine nachweisbaren Spuren zurück. Es verdient vielleicht noch hervorgehoben zu werden, dass anffallende Gerinnselbildungen niemals bei den Sectionen gefunden wurden, und dass das aus den grösseren Gefässen und dem Herzen entleerte Blut in normaler Weise gerann.

Von zwei Versuchen, in welchen das Gift intravenös, bezw. in die Peritonealböble injicirt worden war, lasse ich die Protokolle hier folgen.

1. Hund von 4,75 Kilo. Um 12 h. 2 cem des zweiten Macerates werden in die Vena dorsalis pedis injicirt. Gleich nach der Injection ist das Thier munter, eine Stunde später schon etwas matt.

$3 \mathrm{~h} .15 \mathrm{~m}$. Nachmittags. Erbrechen, ausserdem grosse Mattigkeit.

Die Schwäche nimmt im Laufe des Nachmittages rasch zu. Bauch etwas aufgetrieben. Das Thier ist sehr apathisch und stöbnt. Respiration tief und selten, Herzaction frequent. Auch auf stark schmerzhafte Eingriffe erfolgt nicht die geringste Reaction. Cornealreflex fehlt. Um die Organe möglichst frisch untersuchen zu können wird das sichtlich schon moribunde Thier um $7 \mathrm{~h} .15 \mathrm{~m}$. durch den Nackenstich getödtet.

Sectionsbefund. In der Bauchhöhle circa $150 \mathrm{ccm}$ einer Fluissigkeit von dem Aussehen einer Hämoglobinlösung, dunkelblutroth, nicht gerinnend, mit Wasser klar mischbar und unter dem Mikroskop frei von Blutkörperchen. Gerinnsel finden sich in der Bauchhöhle nur in kleiner Menge in der Umgebung des Pankreas. Peritoneum glatt, frei von Auflagerungen, nicht gerade auffallend geröthet.

Serosa des ganzen Darmes dunkelroth gefärbt, glanzlos, wie gekocht anssehend, keine Blutungen. Leber sehr dunkel, Gallenbl a se prall gefïllt. Galle nicht blutig. Wand der Gallenblase sehr dick, ödematös.

Im Inneren des Darmes von oben bis unten blutrother, dick-schleimiger Inhalt, Schleimhaut gleichmässig dunkelroth. Ebenso verhält sich die Magenschleimhaut, doch ist der schleimige Inhalt des Magens n i c h t blutig gefärbt.

Nieren dunkelroth (mikroskopischer Befund vgl. oben), Milz wenig verändert, ebenso die Lungen. Im Blute wurden noch intra vitam unveränderte rothe Blutkörperchen nachgewiesen, doch ist ibre Zahl entschieden vermindert. Das aus dem Herzen und der Vena cava entleerte Blut gerinnt. Aus dem Cavablut scheiden sich über Nacht 
reichliche Mengen von Hämoglobinkrystallen ab. In den Coagulis finden sich noch normale Blutkörperchen, in dem dariiberstehenden Blute nicht.

2. Kaninchen von 1,55 Kilo. 1,0 ccm der Giftlösung wird in die Peritonealhöhle injicirt. Kurze Zeit nachher ist das Thier schon sebr matt und legt sich auf den Bauch. Nach 2 Stunden stirbt es.

Section. Unter der Haut nichts Abnormes. Bauch stark aufgetrieben. Das parietale Blatt des Peritoneums besonders im kleinen Becken sehr stark geröthet, auch sonst diffuse Röthung des Bauchfelles, aber keine Hämorrbagien. Viscerales Blatt des Peritoneums an der Oberfäche der Gedärme iberall gleichmässig stark injicirt, am Diinndarm an vielen Stellen stecknadelkopfgrosse Echchymosen. In der Bauchhöhle ziemlich viel blutig-seröse Flüssigkeit. Mesenterium stark injicirt, in den Gefässen aber keine Gerinnsel. Der ganze Dünndarm mit Gasen und dünnschleimigen, wenig gallig gefärbten Massen gefüllt, die viele isolirte, Fetttropfen enthaltende Epithelien und zahllose Mikroorganismen enthalten. Milz blauschwarz und vergrössert, Leber ziemlich blass, Nieren gleichmässig hyperämisch; Bla s e leer, Uterus stark hyperämisch.

Dass das Larvengift den Farbstoff der rothen Blutkörperchen, ohne ihn sonst zu verändern, in Lösung überfuihrt, ist schon in der vorhergehenden Abhandlung angegeben worden. Dass diese Wirkung auch im lebenden Thiere statt hat, geht aus den vorstehenden Schilderungen zur Genüge hervor. Bringt man einen Tropfen frisch aus der Obrarterie eines Kaninchens entnommenen Blutes unter ein Deckgläschen und an den Rand derselben einen Tropfen der Giftlösung, so beobachtet man unter dem Mikroskop alsbald eine vollständige Entfärbung der rothen Blutkörperchen. Die Form derselben bleibt zunächst unverändert, erst nach längerer Einwirkung nehmen sie Kugelgestalt an.

Um etwaige directe Wirkungen auf das Protoplasma lebender Zellen nachzuweisen, wurde mikroskopisch das Verbalten der Flimmerepithelien aus der Mundhöhle des Frosches der Giftlösung gegen. über beobachtet. An einzelnen Zellen konnte schon 20-35 Minuten nach dem Hinzutreten des Giftes das Aufhören der Flimmerbewegungen, nach 2 Stunden der Tod aller im Präparate befindlichen Zellen constatirt werden, während die Zellen eines Controlpräparates in physiologischer Kochsalzlösung noch nach 4 Stunden lebhafte Bewegungen zeigten. -

In einer weiteren Versuchsreihe sollten die entzündungserregenden Wirkungen des Larvengiftes genauer. verfolgt werden. Als Beobachtungsobjecte dienten hierbei die Conjunctivalschieimbant und die Ohrmuschel des Kaninchens und der Kamm des Hahnes.

Die Folgen der Einträufelung der wässrigen Lösung des Larven- 
Ueber die Wirkungen des Giftes der Larven von Diamphidia locusta. 441

giftes in den Conjunctivalsack sind aus nachfolgenden Versuchsprotokollen ersichtlich.

1. Ein Tropfen der Lösung wird um $10 \mathrm{~h} .30 \mathrm{~m}$. in den Conjunctivalsack eines weissen Kaninchens gebracht.

12 h. Starke Hyperämie der Conjunctiva.

3. h. Am äusseren Augenwinkel befinden sich dicke Eitertropfen. Die Conjunctiva ist stark ödematös. Das Secret zeigt unter dem Mikroskop Massen unbeweglicher Leukocyten, ausserdem sehr viele in lebhafter Molecularbewegung befindliche Kokken und durchsichtige fettähnliche Tröpfchen. Die Affection hat sich auch auf die Nasenhöble fortgepflanzt, welche lebhaft secernirt.

$6 \mathrm{~h}$. Das Oedem bat am oberen Augenlid noch zugenommen; grosse Mengen milchähnlichen Secretes. Cornea ganz unverändert.

A m folgenden Morgen war die Affection entschieden schon im Rückgange. Die Secretion hat aufgehört, nur das Oedem besteht noch. Das Thier ist ganz munter und frisst. Aus der Nase fliesst noch etwas Secret ab, das genau so wie das Conjunctivalsecret beschaffen ist. A m dritten Tage ist das Auge, von einer leichten Hyperämie abgesehen, wieder normal. Nach 4 Monaten wurde das Thier, das inzwischen nicht weiter beobachtet worden war, zu einem anderen Zwecke getödtet. Bei dieser Gelegenheit fand sicb, dass inzwischen eine totale Atrophie des Bulbus des betreffenden Auges eingetreten war. Der zugehörige Nervus opticus war gleichfalls atrophisch. In der Orbita einige alte Blutergüsse.

2. Die rechtsseitige Conjunctiva eines grossen, munteren Kaninchens wird vorsichtig mit $1 / 2 \mathrm{ccm}$ der frischen Giftlösung bespült.

Bereits nach $30 \mathrm{M}$ in uten starke ödematöse Schwellung, besonders des oberen Lides. Das Auge krampfbaft geschlossen. In einem Strichpräparat des Bindehautsecretes befinden sich Plattenepithelien, Fetttröpfchen und Kokken, noch keine Leukocyten.

Nach 1 Stunde Zunahme des Oedemes, im Secret wenige Leukocyten.

Nach 1 h. $10 \mathrm{~m}$. erscheint das obere Lid kirschgross hervorgewölbt, der Bulbus tief in die Orbita zuriickgedrängt und beim Emporheben des Lides nicht sichtbar; das Secret bereits reicher an Leukocyten, die nach 2 Stunden zu grossen Herden angewachsen sind.

Nach $3 \mathrm{~h} .30 \mathrm{~m}$. beginnt das Oedem abzunehmen, während die Menge des milchig-eitrigen Secretes noch zunimmt. Dasselbe besteht, 4 h. $15 \mathrm{~m}$. nach Beginn des Versuches, fast ausschliesslich aus polynucleären und wenigen grossen mononucleären Eiterkörperchen.

Am darauffolgenden Tage ist das Auge verklebt und lässt sich nur mit Gewalt öffnen. Das Oedem hat zwar bedeutend abgenommen, der Bulbus liegt aber noch tief in der Orbita; im Secret finden sich keine Leukocyten mehr. Im Verlaufe der nächsten 24 Stunden tritt der Bulbus allmählich wieder hervor. Die Cornea ist völlig normal.

Nachdem das Thier noch 2 Tage nach Ablauf der Conjunetivitis sich scheinbar ganz wohl befunden hatte, traten 6 mal 24 Stunden nach der Einträufelung Vormittags $11 \mathrm{~h}$. ohne Vorboten epileptiforme Krämpfe auf. Das Thier streckt die Extremitäten von sich und wirft sich von 
einer Seite auf die andere; 20 Secunden nach dem ersten Krampfanfall liegt es ruhig auf der Seite, als ob es verenden wollte, richtet sich aber noch einmal auf und vermag, auf den Fussboden gesetzt, durch Nachziehen der Hinterbeine sich schwerfällig fortzubewegen. Da der Verdacht auf eine pathologische Veränderung des Augenhintergrundes nahe lag, wird das Thier gespiegelt; die Untersuchnng ergiebt indess hier nichts Abnormes. Während des Spiegelns, um 12 h. verfällt das Kaninchen aber von Neuem in Krämpfe, die sich in kurzen Zwischenräumen wiederholen und um $12 \mathrm{~h} .34 \mathrm{~m}$. zum Tode führen. Bis kurz vor dem Tode waren Lid- und Pupillarreflex vorhanden, dagegen war vor einem um $12 \mathrm{~h} .20 \mathrm{~m}$. erfolgten Anfall eine Lähmung des Rectus superior des $\mathrm{rech}$ ten Auges zu constatiren, der Bulbus stark nach unten rotirt und hervorgetrieben.

Die sogleich vorgenommene Obduction ergab Hyperämie der Hirnhäute und Sinus. An der Eintrittsstelle des Opticus finden sich beiderseits offenbar frische, erbsengrosse Blutergiisse. Am Opticus, Bulbus und im Gehirn lässt sich ebenso wie in den übrigen Organen weiter nichts Pathologisches nachweisen.

Beide Versuche zeigen übereinstimmend, dass das Larvengift analog wie das Abrin der Jeguiritisamen eine typische acute Conjunctivitis erzeugt, wenn es in den Conjunctivalsack gebracht wird, ohne dass hierbei, auch bei der Anwendung grösserer Giftmengen, wie sie absichtlich im zweiten Versuche stattfand, die charakteristischen Allgemeinwirkungen - Abnahme der Fresslust und Hämoglobinurie auftreten. Man darf hieraus folgern, dass die Schleimhautepitbelien die Resorption des Giftes verhindern, und dass dasselbe wahrscheinlich durch die alsbald einsetzende mächtige Leukocytenemigration zum grössten Theil unschädlich gemacht wird.

Die Genese des in beiden Fällen, im ersteren sehr chronisch, im letzteren verhältnissmässig acut sich anschliessenden Uebergreifens der Wirkung auf den Bulbus, bezw. das centrale Nervensystem wird sich nur durch weitere Versuche befriedigend aufklären lassen.

Beobachtungen an Kaninchenohr versprachen die Möglichkeit, die versehiedenen Stadien des Entzündungsprocesses in ihrer Aufeinanderfolge beobachten zu können. Die Untersuchung wurde folgendermaassen ausgeführt. An beiden Obren eines womöglich weissen Kaninchens werden die Haare entfernt, hierauf das für die Giftapplication ausersehene durch leichtes Einklemmen in die mit Kork ausgelegten Branchen eines Holzstatives in senkrechter Stellung gehalten, so dass es gut im durchfallenden Tageslichte beobachtet werden kann. Nachdem die durch die Vorbereitungen verursachte Hyperämie des Ohres verschwunden war, wurden mittels einer sehr feinen Injectionsnadel $0,1-0,2 \mathrm{cem}$ der Giftlösung an einer von grösseren Gefäss- 
Ueber die Wirkungen des Giftes der Larven von Diamphidia locusta. 443

zweigen freier Stelle an der Aussenseite zwischen Haut und Ohrknorpel injicirt.

1. 19. Mai. 9 h. $30 \mathrm{~m}$. Injection von $0,2 \mathrm{ccm}$ einer $z$ weiten Maceration ziemlich in der Mitte des Ohres. Unmittelbar darauf erscheint das Ohr in der Umgebung der Injectionsstelle blass; eine grosse Vene am Rande der Ohrmuschel aber innerhalb des Injectionsbezirkes blutle er, oberhalb und unterhalb des Injectionsbezirkes hingegen prall gefüllt. Die Fuillung des leeren Abschnittes kann auch durch eentrale Compression der Vene nicht wieder hergestellt werden.

10 b. 20 m. Die Randvene des Ohres innerhalb des Injectionsbezirkes noch ganz leer.

$10 \mathrm{~h} .40 \mathrm{~m}$. Die Vene hat sich bis auf ein $2 \mathrm{~mm}$ langes Stiick in der Nähe der Einstichsstelle wieder mit Blut gefullt. In der Näbe der Venenverzweigungen des Injectionsbezirkes tritt eine verwaschene hellrosarothe Färbung auf.

$11 \mathrm{~h}$. Die Röthung hat zugenommen. Ein kleines Stiückchen der Randvene ist immer noch blutleer. Das ganze injicirte $\mathrm{Ohr}$ entschieden röther und wärmer als das gesunde.

12 h. Die Röthung hat noch mehr zugenommen. Im Bereiche der Injectionsstelle sind nunmehr auch die vorher kaum sichtbaren Arterien am Rand und in der Mitte stark gefillt zum Vorschein gekommen. Um die Venen herum überall hellrothe, verwaschene Streifen.

4 b. Die Röthung hat sich eoncentrisch um den Injectionsort um etwa das $11 / 2$ fache der anfänglichen Ausdehnung ausgebreitet. Das ganze Ohr ist geschwollen (ödematös). Die Gefässe im Entzündungsherd kaum mehr durchscheinend.

6 h. $30 \mathrm{~m}$. Derselbe Befund.

20. Ma i Morgens. An der Innenseite des Ohres ist an zwei Stellen die Epidermis durch eine braunrothe Flüssigkeit blasig abgehoben. An der Aussenseite in der Nähe der Einsticbstelle entleert sich ein Tropfen zäher Eiter, in welchem unbeweglich Leukocyten, viel feinkörniger Detritus und viele stark glänzende Kerne zu erkennen sind. Schwellung und Infiltration des Ohres noch unverändert. Aus der Epidermisblase mit einem Capillarröhrehen etwas hellbraune durehsiehtige Flüssigkeit entnommen, worin körperliche Elemente mit Ausnahme kleinster Protoplasmaklümpehen nieht zu erkennen sind.

21. Mai. Die Schwellung hat am Rande des Ohres etwas abgenommen. Die Epidermisblasen an der Innenseite haben sich verbreitert. An der Einstichsstelle Borkenbildung; Gefässe im Entzündungsherd immer noch nicht durchscheinend.

22. Mai. An der Innen- und Aussenseite des Ohres wird dicker, zäher Eiter abgesondert. Die Leakocyten ohne Bewegung, sehr körnchenreich; ansserdem enthält der Eiter Epidermiszellen und elastische Fasern.

23. Mai. Am Rande des Entzündungsherdes nach innen hat sich ein scharfer, etwas erhabener Demarcations wall gebildet. Das Thier, wie bisher, ganz munter.

24. Mai. Demareation sehreitet fort. Im Centrum des Herdes beginnt 
das Gewebe einzutrocknen. In der Peripherie um den Herd starke Gefässinjection.

7. Juni. A m 20. Tage nach der Injection. Der Schorf hat sich abgestossen. Im Ohr ein ovales Loch von der Grösse eines Einmarkstïckes, das von gut geheilten und überhänteten, abgerundeten Rändern umgeben ist. Das Thier, noch lange beobachtet, bleibt gesund.

2. $0,2 \mathrm{ccm}$ einer frischen ersten Maceration zwischen zwei grösseren Gefässen injicirt.

Nach $1 \mathrm{~h} .15 \mathrm{~m}$. tritt in der Nähe einer grösseren Vene eine verwaschene hellrothe Färbung auf, die nach $2 \mathrm{~h} .45 \mathrm{~m}$. eine pfennigstitekgrosse Fläche einnimmt. $4 \mathrm{~h} .15 \mathrm{~m}$. nach der Injection sind sämmtliche Gefässe stark injicirt; das Capillarnetz gut sichtbar. Die diffuse Röthung hat zugenommen und erstreckt sich von der Injectionsstelle bis an die Ohrwurzel.

Nach 8 Stunden starke Röthung und Schwellung des ganzen Ohres. An der Innenseite sickert ein blutroth gefärbtes seröses Fluidum ans, das nur vereinzelte Lenkocyten nnd keine rothen Blutkörperchen enthält.

Nach $15 \mathrm{Stunden} 30 \mathrm{ccm}$ dunkelbutrother Harn entleert.

Nach 24 Stunden. Das sebr stark ödematöse Ohr hängt schlaff herab. Die Abscheidung blutigserösen Exsudates an der Innenseite hat zugenommen. Nach $40 \mathrm{Stu}$ uden abermals Entleerung blutrothen Harnes.

Nach 48 Stunden. Tod des Thieres.

Sectionsb efund. Blutig-ödematöse Infiltration des Unterhautzellgewebes erstreckt sich bis in die unteren Halspartien. Nierenbefund wie oben beschrieben. Mikroskopische Untersuchung des Ohres ergiebt diffuse Infiltration mit Rundzellen, die an einzelnen Stellen des ödematösen Gewebes in grossen Massen angebäuft sind.

3. 0,1 cem einer zweiten Maceration injicirt.

Nach 5 Minuten erscheint das Lumen der den Injectionsbezirk durchziehenden Venen blutleer.

Nach 15 Minuten beginnt die Entstehung eines rothen Hofes um die Injectionsstelle. An beiden Seiten der Venenstämme erscheinen blassrothe Bänder.

Nach $2 \mathrm{St}$ tund en starke Injection aller Gefässe. Die mittlere Hauptvene erscheint als ein $3 \mathrm{ccm}$ breites, diffus rothgefärbtes Band.

Nach 24 Stunden wird das Ohr behufs mikroskopiseher Untersuchung amputirt, parallel mit der Querachse in Streifen zerlegt und diese sofort fixirt.

Die histologischen Bilder zeigen eine Anhäufung von Rundzellen, insbesondere in der Umgebung der stark gefüllten Venen. In letzteren selbst sieht man in der Peripherie des Lumens eine grössere Zahl von Leukocyten als in den normalen Gefässen von Controlpräparaten.

Ueber die Wirkung des Larvengiftes auf das Gewebe des $\mathrm{Hab}$ nenkammes giebt folgendes Protokoll Aufsehluss.

Einem gesunden $\mathrm{Hahn}$ werden $0,4 \mathrm{ccm}$ einer Normalmaceration in Jie Mitte des Kammes in der Richtung von vorn nach hinten injicirt. 
Ueber die Wirkungen des Giftes der Larven von Diamphidia locusta. 445

Sofort entsteht eine etwa bohnengrosse blutleere Stelle von weisser Farbe and scharfer Begrenzung.

Nach 15 Minuten färbt sich der ganze hintere Theil des Kammes blauroth (Stase?), wobei zugleich die Begrenzung des blutleeren Bezirkes nach hinten etwas verwaschen erscheint, wärend die vordere Kammhälfte ihre normale lebhafte Färbung behält.

70 Minuten nach der Injection beginnt die weisse, blutleere Zone sich allmählich livide bläulich roth zu färben, während auch die hintere Kammhälfte wieder hellroth wird.

Nach 24 Stunden bat sich um den Injectionsbezirk eine scharfe Demarcationslinie von dunkelblauer Farbe gebildet, und nach weiteren 24 Stunden (nach 2 Tagen) das Gewebe im Bereich der Injection in der Ausdehnung eines Zehnpfennigstückes sich graugrünlich gefärbt, stark zusammengezogen und scharf gegen das umgebende lebhaft rothe, normale Gewebe abgegrenzt.

Nach 3 Tagen beginnt das betreffende Stïck nekrotisch zu werden, olune dass an der Oberfäche ein Tropfen Flüssigkeit austritt. Der Herd lockert sich im Laufe der nächsten Tage, wird aber erst am 17. Tage nach der Injection als bohnengrosser Schorf abgestossen, so dass der Kamm in der Mitte ein mit gut geheiltem Rand umgebenes Loch zeigt. Das Thier verhielt sich zwar während des Ablaufes des Processes ruhiger als vorher, uberlebte jedoch den Versuch.

Der Verlauf der Localwirkung beim Hahne unterscheidet sich insofern von den bei Kaninchen wahrgenommenen Erscheinungen, als hier kein Oedem, keine sichtbare Eiterung, kein Uebergreifen der Entzïndung auf die Umgebung eintrat, der Process vielmehr den Charakter einer trockenen Nekrose hatte.

Ich habe dann endlich auch noch in einfacher Weise mich von der chemotaktischen Wirkung des Larvengiftes auf Leukocyten überzeugen können. Feine Capillarröhrchen von $4 \mathrm{~cm}$ Länge wurden mit der Giftlösung gefüllt, an beiden Enden zugeschmolzen und in Alkohol keimfrei gemacht. Davon wurden 3 Stück unter aseptischen Cautelen einem erwachsenen Kaninchen unter die Haut am Rücken, an der rechten und linken Bauchseite gebracht. Nach 24 Stunden wurde ein Röhrchen, nach 2 Tagen die beiden anderen subcutan zerbrochen. Die nach einigen Tagen durch Einschnitte wieder zu zu Tage geförderten Röhrenstiicke enthielten sämmtliche an beiden Enden weissliche Pfröpfe, welche, wie das Mikroskop erwies, nur aus Leukocyten bestanden. -

Die Beobachtungen an der Conjunctiva und am Ohr des Kaninchens machen es unzweifelhaft, dass wir in dem Larvengift einen Stoff besitzen, der in typischer Form den Symptomencomplex der Entztindung hervorruft. Durch diese seine Wirkung erklären sich auch die oben beschriebenen Veränderungen, welche nach subcutaner 
446 XXVIII. Starcke, Wirkungen des Giftes der Larven von Diamphidia locusta.

Injection des Giftes in weiter Ausdehnung rom Applicationsorte aus in den anliegenden Geweben sich darbieten.

In zwei Punkten unterscheidet sich diese Wirkung von der anderer entzündungserregender Agentien. Einmal darin, dass im Entzündungsgebiete, wie obige Versuche am Kaninchenohr zeigen, schon kurze Zeit nach dem Beginne der Wirkung des Entzündungsreizes gelöster Blutfarbstoff auftritt. Bei der Injection unter die Haut des Rumpfes kommt dadurch offenbar die intensiv blutrothe Färbung des ödematös geschwollenen Gewebes zu Stande. Der zweite Unterschied besteht darin, dass die Wirkung des Entzindungsreizes sich auf weite Strecken fortpflanzt, wenn das Gift in das subcutane Zellgewebe gelangt. Auch vom Kaninchenohr aus haben wir in einem Falle die Wirkung bis in die Halsgegend sich fortpflanzen sehen. In den anderen Fällen war es wohl infolge der Verwendung einer schwächeren Giftlösung gelungen, die Entzỉndung auf einen engeren Raum einzuschränken. Jene weite Verbreitung der entzündlichen Wirkung spricht dafür, dass das hier in Frage stehende Gift mit dem Lymphstrom sich auf weitere Entfernungen unverändert verbreiten kann, während andere chemische Entzündungsreize infolge ihrer chemischen Eigenschaften durch eine directe Veränderung des Gewebes an der Applicationsstelle zurückgehalten oder doch wenigstens nicht weit über dieselbe hinaus unverändert forttransportirt werden.

Auf einen Vergleich der Wirkung des Larvengiftes mit der anderer Toxalbumine soll hier nicht näher eingegangen werden. Trotz manchen Analogien und Aehnlichkeiten stimmt es mit keinem derselben ganz überein.

Die charakteristischen Züge der Wirkung des Larvengiftes sind die Lösung des Blutfarbstoffs und die Erregung von Entzündung. Die Symptome der Vergiftung während des Lebens und die Leichenbefunde sind zum grössten Theil und ungezwungen auf diese beiden Wirkungen zurückzuführen. Die schweren Entzündungsvorgänge nnter der Haut erklären das langsame Dahinsiechen der Thiere bei mehr chronischem Verlauf der Vergiftung, der mit der Hämoglobinauflösung unzertrennlich verknüpfte Untergang eines mebr oder weniger grossen Theiles der rothen Blutlörperchen den raschen tödtlichen Verlauf in acuten Fällen. Hinsichtlich der centralnervösen Symptome bei acuter Vergiftung mag es dahingestellt bleiben, in wieweit sie von der Blutveränderung oder von einer specifischen Einwirkung des Giftes auf die Nervenzellen abhängig sind. 